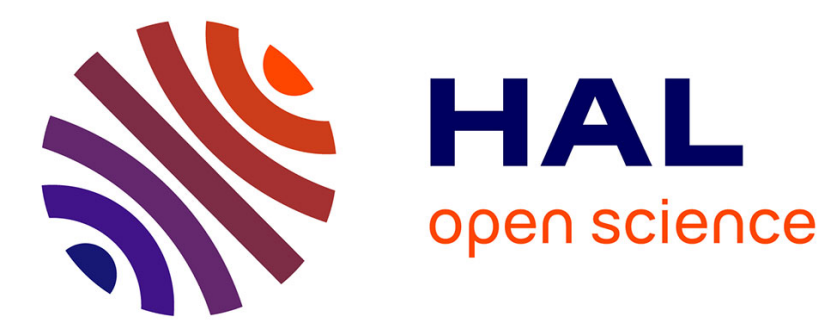

\title{
Control of the Mechanical Adhesion of III-V Materials Grown on Layered h-BN
}

Phuong Vuong, Suresh Sundaram, Adama Mballo, Gilles Patriarche, Stefano

Leone, Fouad Benkhelifa, Soufiane Karrakchou, Tarik Moudakir, Simon

Gautier, Paul L Voss, et al.

\section{To cite this version:}

Phuong Vuong, Suresh Sundaram, Adama Mballo, Gilles Patriarche, Stefano Leone, et al.. Control of the Mechanical Adhesion of III-V Materials Grown on Layered h-BN. ACS Applied Materials \& Interfaces, 2020, 12 (49), pp.55460 - 55466. 10.1021/acsami.0c16850 . hal-03350674

\section{HAL Id: hal-03350674 \\ https://hal.science/hal-03350674}

Submitted on 21 Sep 2021

HAL is a multi-disciplinary open access archive for the deposit and dissemination of scientific research documents, whether they are published or not. The documents may come from teaching and research institutions in France or abroad, or from public or private research centers.
L'archive ouverte pluridisciplinaire HAL, est destinée au dépôt et à la diffusion de documents scientifiques de niveau recherche, publiés ou non, émanant des établissements d'enseignement et de recherche français ou étrangers, des laboratoires publics ou privés. 


\title{
Control of the Mechanical Adhesion of III-V Materials Grown on Layered h-BN
}

\author{
Phuong Vuong, Suresh Sundaram, Adama Mballo, Gilles Patriarche, Stefano Leone, Fouad Benkhelifa, \\ Soufiane Karrakchou, Tarik Moudakir, Simon Gautier, Paul L. Voss, Jean-Paul Salvestrini, \\ and Abdallah Ougazzaden*
}

\begin{abstract}
Hexagonal boron nitride (h-BN) can be used as a p-doped material in wide-bandgap optoelectronic heterostructures or as a release layer to allow lift-off of grown three-dimensional (3D) GaN-based devices. To date, there have been no studies of factors that lead to or prevent lift-off and/or spontaneous delamination of layers. Here, we report a unique approach of controlling the adhesion of this layered material, which can result in both desired lift-off layered h-BN and mechanically inseparable robust h-BN layers. This is accomplished by controlling the diffusion of $\mathrm{Al}$ atoms into h-BN from AlN buffers grown on h-BN/sapphire. We present evidence of $\mathrm{Al}$ diffusion into h-BN for AlN buffers grown at high temperatures compared to conventional-temperature AlN buffers. Further evidence that the $\mathrm{Al}$ content in $\mathrm{BN}$ controls lift-off is provided by comparison of two alloys, $\mathrm{Al}_{0.03} \mathrm{~B}_{0.97} \mathrm{~N} /$ sapphire and $\mathrm{Al}_{0.17} \mathrm{~B}_{0.83} \mathrm{~N} /$ sapphire.

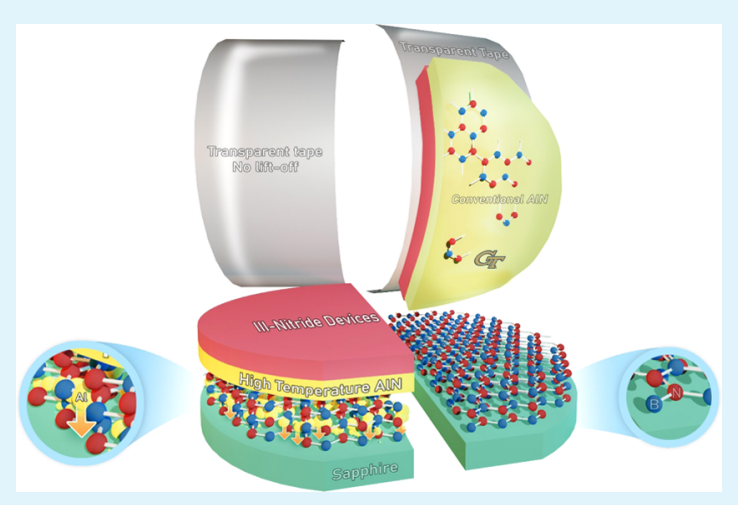
Moreover, we tested that management of $\mathrm{Al}$ diffusion controls the mechanical adhesion of high-electron-mobility transistor (HEMT) devices grown on AlN/h-BN/sapphire. The results extend the control of two-dimensional (2D)/3D hetero-epitaxy and bring h-BN closer to industrial application in optoelectronics.
\end{abstract}

KEYWORDS: 2D boron nitride, III-nitrides, semiconductors, mechanical transfer, flexible (opto) electronics, transferrable nanodevices

\section{INTRODUCTION}

Optoelectronic and electronic applications of hexagonal boron nitride (h-BN), a two-dimensional (2D) ultrawide-bandgap semiconductor, have attracted extensive attention in the last decade. Van der Waals (vdW) epitaxial growth of 2D h-BN layers and subsequent three-dimensional (3D) III-nitride device structures-based h-BN has been demonstrated. ${ }^{1-7}$ Kobayashi et al. demonstrated the successful transfer of GaN-based blue light-emitting diodes (LEDs) from h-BN onto foreign substrates using h-BN as a mechanical release layer. ${ }^{1}$ A goal of subsequent research was the wafer-scale transfer of III-N devices to flexible substrates. Transfers of different device structures such as $\mathrm{AlGaN} / \mathrm{GaN}$ high-electronmobility transistors (HEMTs), ${ }^{2,6} \mathrm{AlGaN} / \mathrm{GaN}$-based gas sensors, ${ }^{4}$ InGaN/GaN multi-quantum-well LEDs, and solar cells $^{8-10}$ have been then demonstrated at the wafer scale.

Ultrawide-bandgap (UWBG) h-BN also reveals a new generation of integration between $2 \mathrm{D}$ materials and bulk materials for physical coupling, such as deep ultraviolet (DUV) sources, ${ }^{11,12}$ neutron detectors, ${ }^{13,14}$ and a hybrid III-N/2D ultraviolet (UV) photodetection. ${ }^{15,16}$ In these cases, the h-BN layer should adhere to the neighboring layers. However, it has been challenging to control spontaneous delamination and the degree of force needed for lift-off of III-N device hetero- structures on h-BN. ${ }^{13,17-19}$ Researchers have reported spontaneous delamination of h-BN during growth owing to the mismatch in the thermal expansion coefficient between h$\mathrm{BN}$ and substrates during cooldown. ${ }^{19,20}$ The delamination of $\mathrm{h}-\mathrm{BN}$ is also observed during front-end device processing with some liquids during cleaning steps. ${ }^{13,19}$ These issues influence the performance of the devices and may ultimately block the route to large-scale commercial device fabrication on h-BN.

An essential component of III-N on h-BN is the use of aluminum-based buffer layers on h-BN for growth of highquality GaN device structures. ${ }^{21}$ It has been demonstrated that an AlN buffer greatly improves the surface morphology and crystalline quality of subsequent nitride layer growth, compared to an AlGaN buffer. ${ }^{22-25}$ However, the conflict in the mechanical lift-off of AlN/h-BN structures emerges, where the lift-off process is not always successful. ${ }^{24}$ Further research

Received: September 18, 2020

Accepted: November 16, 2020 


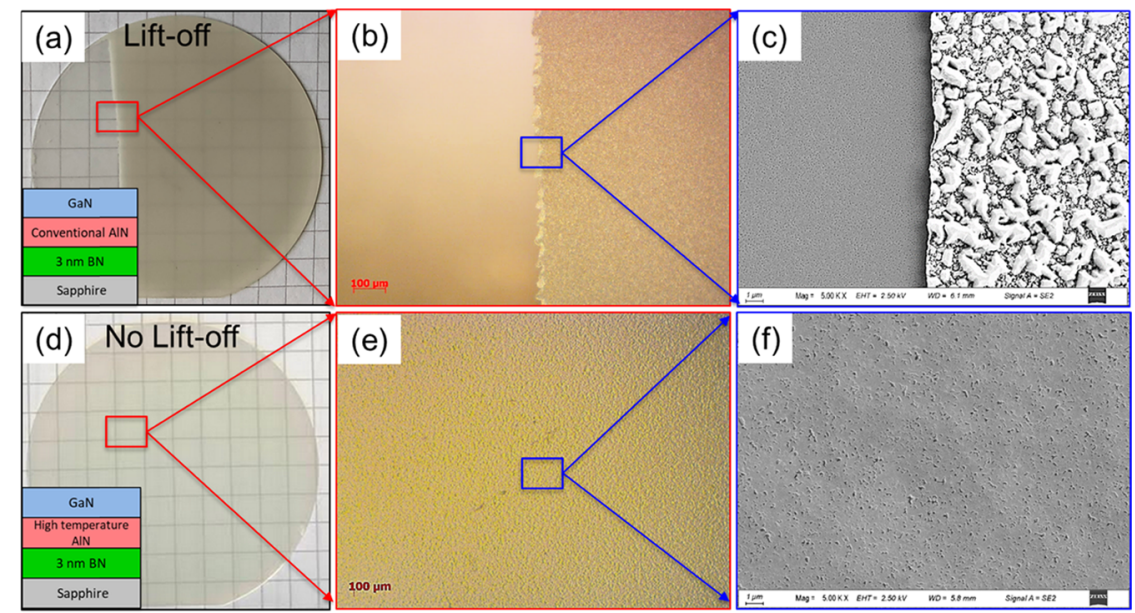

Figure 1. Photograph, optical microscope, and SEM images of GaN/conventional AlN/BN (a-c) and GaN/high-temperature AlN/BN (d-f) grown on sapphire, respectively. Insets of (a) and (d): schematic illustration of the grown structures.
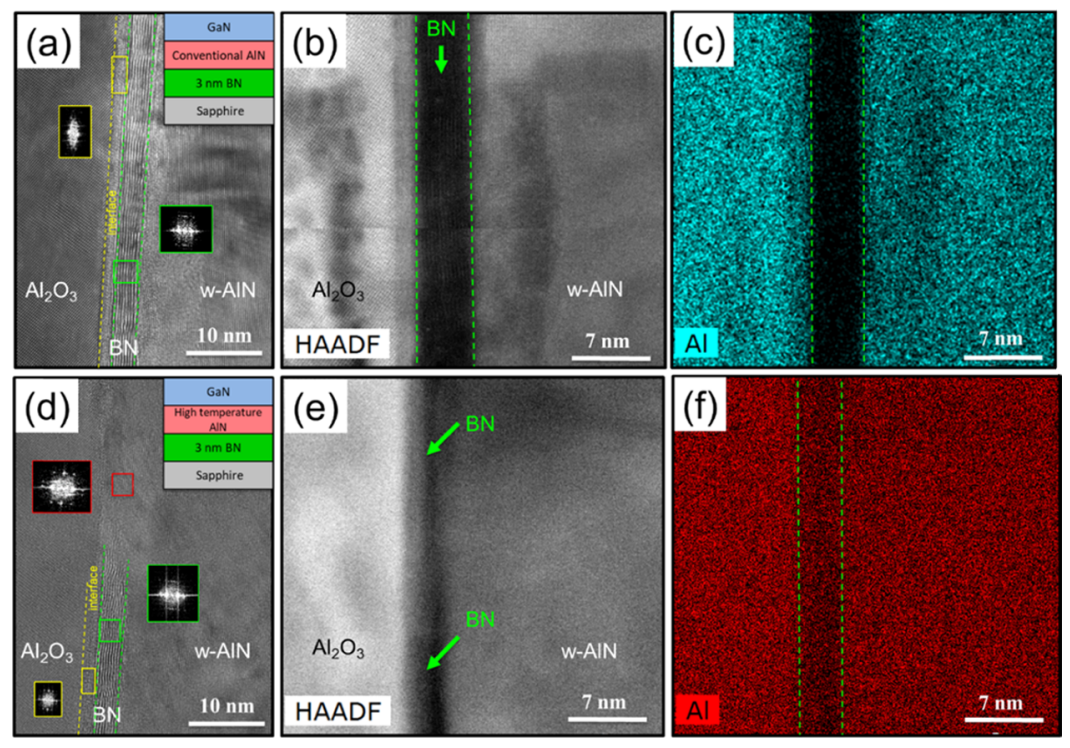

Figure 2. Cross-sectional high-resolution TEM and bright-field HAADF-STEM images, and energy-dispersive X-ray spectroscopy (EDX) elemental mappings of $\mathrm{Al}$ of GaN/conventional AlN/h-BN $(\mathrm{a}-\mathrm{c})$ and $\mathrm{GaN} /$ high-temperature AlN/h-BN $(\mathrm{d}-\mathrm{f})$ grown on sapphire, respectively. Insets of $(\mathrm{a})$ and (d): schematic illustration of the grown structure.

on this topic is required to clearly understand the mechanism and conditions needed for lift-off.

In this article, we report a growth technique that eliminates spontaneous delamination and prevents lift-off of 3D III-N layers grown on $\mathrm{h}-\mathrm{BN}$. This is accomplished by controlling the diffusion of $\mathrm{Al}$ atoms into h-BN from AlN buffers grown on thin ( $<5 \mathrm{~nm}$ thick) h-BN on sapphire substrates. First, we study mechanical lift-off of GaN/AlN/h-BN structures with AlN layer grown conventionally $\left(1100{ }^{\circ} \mathrm{C}\right)$ and at a high temperature $\left(1280{ }^{\circ} \mathrm{C}\right)$. Detailed structural analysis of these samples was performed using high-angle annular dark-field scanning transmission electron microscopy (HAADF-STEM) and energy-dispersive X-ray spectroscopy (EDX) measurements, allowing for characterization of crystal structure and $\mathrm{Al}$ content. We also test lift-off with two BAlN alloys, $\mathrm{Al}_{0.03} \mathrm{~B}_{0.97} \mathrm{~N} /$ sapphire and $\mathrm{Al}_{0.17} \mathrm{~B}_{0.83} \mathrm{~N} /$ sapphire. Then, we test lift-off and measure $\mathrm{Al}$ content in $3 \mathrm{~nm}$ thick h-BN and $25 \mathrm{~nm}$ thick h-BN topped with high-temperature AlN to show the role of diffusion. Finally, we tested that management of $\mathrm{Al}$ diffusion controls the mechanical adhesion of HEMT devices grown on $\mathrm{AlN} / \mathrm{h}-\mathrm{BN} /$ sapphire.

\section{RESULTS AND DISCUSSION}

We first address the mechanical lift-off of the GaN/AlN/h-BN structures grown on sapphire with different growth temperatures of the AlN layer. One set is grown at $1100{ }^{\circ} \mathrm{C}$ (conventional AlN). The second set is grown at $1280{ }^{\circ} \mathrm{C}$ (high-temperature AlN). The mechanical lift-off comparison between the two sets of samples is presented in Figure 1, which involves camera photograph, optical microscope, and scanning electron microscope (SEM) images. The GaN/conventional AlN/h-BN structure enables to lift from their substrates using a transparent scotch tape, as seen in Figure 1a-c. A high contrast between lifted and no-lifted areas is clear from the camera photograph and optical microscope (Figure 1a,b). The SEM image clearly shows the three-dimensional (3D) morphology of the top GaN layer compared to the flat surface of the released area (Figure 1c). On the other hand, using the 


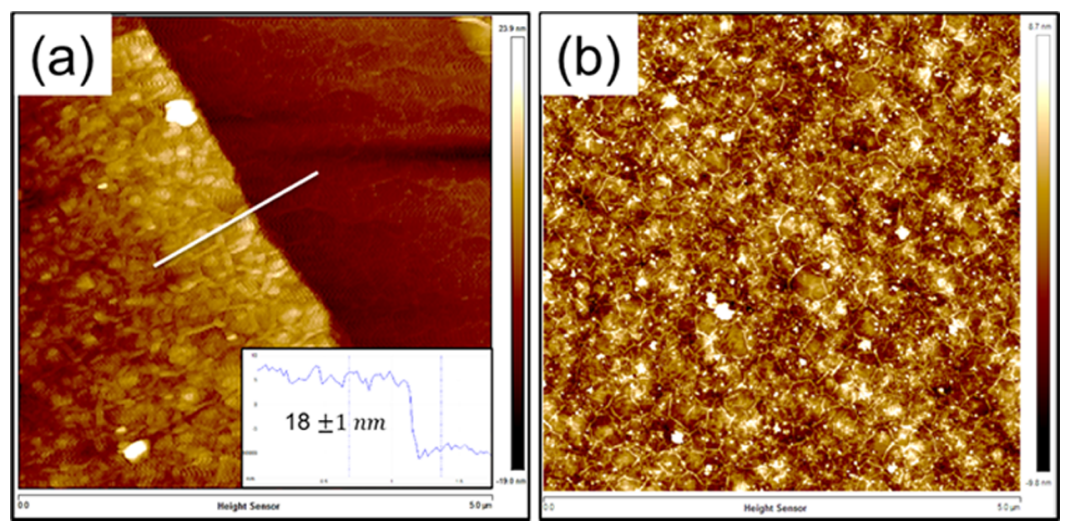

Figure 3. Atomic force microscopy (AFM) images for the BAlN alloys grown on sapphire after lift-off with different $\mathrm{Al}$ contents: (a) 3.5\% and (b) $17 \%$.

same procedure with scotch tape, the lift-off of the GaN/hightemperature AlN/h-BN structures was not possible despite several attempts. Figure $1 \mathrm{~d}-\mathrm{f}$ presents photograph, optical microscope, and SEM images showing the uniform surface of the sample after lift-off attempts. The improved morphology smoothness of the GaN layer compared to the previous case is due to the better quality of the buffer layer with improved AlN grown at high temperatures, as demonstrated in refs 1, 22, 26. The high-resolution X-ray diffraction (HR-XRD) scans of the two sets of samples are shown in Section 1 in the Supporting Information. These adhesion behaviors of h-BN as a function of buffer layer has never been reported and raises questions about the role of AlN layer in the lift-off mechanism of III-V structures.

Detailed structural analyses of the conventional (Figure 2a) and high-temperature (Figure 2d) AlN on h-BN were performed using transmission electron microscopy (TEM), showing different areas of interest in the structures. In two sets of samples, a two-dimensional (2D) h-BN layer with the lattice oriented along the $c$-axis was observed. The insets of Figure $2 \mathrm{a}, \mathrm{d}$ present the fast Fourier transform (FFT) pattern, corresponding to the selected areas (green boxes), identifying clearly a single-crystalline hexagonal phase. The interlayer distance of the h-BN layers is calculated to be $0.349 \pm 0.003$ $\mathrm{nm}$, which is larger than the reported value $\left(d=0.33 \mathrm{~nm}^{28}\right)$. This $5.7 \%$ increase can be attributed to residual strain.

However, the h-BN layer of conventional AlN/h-BN structures is found to be continuous in contrast to the discontinuous h-BN layer of high-temperature AlN/h-BN structures. In the second set of samples, we detect the presence of w-AlN within the h-BN layer, as proved by the FFT (inset of Figure $2 \mathrm{~d}$-red box), and the calculation of interlayer distance $\left(\sim 0.25 \mathrm{~nm}\right.$-comparable with the reported value $\left.{ }^{29}\right)$. A possible interpretation is that $\mathrm{Al}$ diffusion into the h-BN layer causes the discontinuous h-BN layer as well as the w-AlN inclusions. Previous studies reported that high growth temperature of $\mathrm{AlN}\left(>1100{ }^{\circ} \mathrm{C}\right)$ causes $\mathrm{Al}$ diffusion into other materials such as $\mathrm{GaN}$ or GaAs. ${ }^{30-34}$ In particular, Haneda et al. have demonstrated that the diffusion coefficient of $\mathrm{Al}\left(D_{\mathrm{Al}}\right)$ is increased by 2 orders of magnitude when the growth temperature is increased by $100{ }^{\circ} \mathrm{C} .{ }^{34}$ In our study, high-temperature $\mathrm{AlN} / \mathrm{h}-\mathrm{BN}$ structures, which were grown at $1280{ }^{\circ} \mathrm{C}$ (almost $200{ }^{\circ} \mathrm{C}$ higher than conventional AlN/BN structures), enable $\mathrm{Al}$ atoms to have more kinetic energy and diffuse into h-BN layers, increasing the $\mathrm{Al}$ concentration there. The decomposition rate of AlN is very low at temperatures below $1300{ }^{\circ} \mathrm{C}$, as reported in ref 31 . Therefore, in our study, the $\mathrm{Al}$ diffusion into h-BN layer may occur from the atomic $\mathrm{Al}$ on the surface during the AlN growth and not from the AlN layer. We speculate that the diffused $\mathrm{Al}$ atoms create some bonding between $\mathrm{Al}$ and h-BN, changing the structure of h-BN and $\mathrm{h}-\mathrm{BN} /$ sapphire interface. In some areas, with a high $\mathrm{Al}$ concentration, a w-AlN layer can be formed, as seen in Figure 2d. w-AlN inclusions may act as a "glue", which anchors the hBN layer and thus the full structure on the substrate disabling the mechanical lift-off and any delamination during processing. In the same way, thick high-temperature AlN will lead to longer growth, and more time consequently leads to more $\mathrm{Al}$ atoms diffusing into the h-BN layer, thus making lift-off more difficult. On the other hand, the mechanical lift-off of IIInitride devices on $2 \mathrm{D} \mathrm{h}-\mathrm{BN}$ on the AlN template with successful lifting was reported in refs 5, 27. To control the liftoff of such a structure, AlN should be subjected to temperatures higher than $1300^{\circ} \mathrm{C}$ for $\mathrm{Al}$ to diffuse into h-BN.

In addition, it is interesting to note that the sapphire/h-BN interfaces between the two sets of samples are different. For the conventional AlN/h-BN structures, a 1-2 nm amorphous AlOxN layer is observed, as identified by the FFT pattern (Figure 2a-yellow box). A similar observation has been mentioned and studied by Amano's group. ${ }^{35}$ Conversely, the high-temperature AlN/h-BN structures exhibit a clearly ordered crystalline arrangement of a few w-AlN layers (2-3 $\mathrm{nm}$ in thickness) at the interface of the layered $\mathrm{h}-\mathrm{BN}$ and sapphire substrate (Figure 2d). The diffraction pattern (inset of Figure $2 \mathrm{~d}$-yellow box) and the $0.25 \mathrm{~nm}$ of interlayer distance indicate the wurtzite crystal structure of this AlN layer. We believe that the AlN at the interface sapphire/h-BN is due to the nitridation and its structural quality probably deeply depends on the growth temperature of the above structure, which will need further study to confirm.

To better understand the different impact of the AlN growth temperature on the AlN/h-BN structures, the complementary cross-sectional high-angle annular dark-field scanning transmission electron microscopy (HAADF-STEM) image and the energy-dispersive X-ray spectroscopy (EDX) element mappings of Al have been performed for conventional AlN growth (Figure 2b,c) and for high-temperature AlN growth (Figure $2 \mathrm{e}, \mathrm{f})$. The $\mathrm{Al}$ concentration in the $3 \mathrm{~nm}$ h-BN region of the high-temperature $\mathrm{AlN} / \mathrm{h}-\mathrm{BN}$ structure is 19 atom \%, whereas the $\mathrm{Al}$ concentration of the h-BN region in the conventional sample is less than 3 atom \%. This provides strong support that temperature-dependent diffusion of $\mathrm{Al}$ explains the $\mathrm{Al}$ content 
in the h-BN region. It also provides direct proof for the role of $\mathrm{Al}$ concentration in the lift-off process. At a low $\mathrm{Al}$ concentration, 2D-layered h-BN still exhibits a uniform crystalline structure that enables the mechanical lift-off of the $\mathrm{GaN} /$ conventional AlN/h-BN structures. When the $\mathrm{Al}$ concentration is increased to the critical value, some bonding between $\mathrm{Al}, \mathrm{B}$, and $\mathrm{N}$ can be created and the layered structure of $\mathrm{h}-\mathrm{BN}$ has been changed.

We have recently studied $2 \mathrm{D}$ boron-rich BAlN alloys and found phase separation for an $\mathrm{Al}$ content above $17 \%$ in the presence of wurtzite Al-rich BAlN phases in a matrix of layered hexagonal B-rich BAlN together with the pure w-AlN. ${ }^{36}$ Checking lift-off in BAlN alloys would be expected to confirm that the presence of aluminum content would prevent lift-off and delamination. We examined the mechanical lift-off of single hexagonal phase boron-rich BAlN alloys with $\mathrm{Al}$ contents of 3.5 and $17 \%$. The $\mathrm{Al}$ content was estimated by secondary-ion mass spectrometry (SIMS), as reported in ref 36. We found that only BAlN alloys with $3.5 \% \mathrm{Al}$ could be lifted off, whereas samples with an average $\mathrm{Al}$ content of $17 \%$ could not be lifted off. Figure 3 displays the atomic force microscopy (AFM) images of these alloys after lift-off. The thickness difference between the lift-off part and the non-liftoff part of $3.5 \% \mathrm{Al} \mathrm{BAlN}$ alloys was measured to be $18 \pm 1 \mathrm{~nm}$. The morphology of these alloys exhibits honeycomb-like wrinkles on the surface, which is comparable to the standard morphology of un-alloyed $2 \mathrm{D} \mathrm{h-BN}{ }^{37}$ In additional to wrinkles, particles (white spots) were observed on the surface of the two samples (more pronounced in $17 \% \mathrm{Al}$ BAlN alloys), confirming the $\mathrm{Al}$ incorporation. When the presence of $\mathrm{Al}$ in h$\mathrm{BN}$ is high $(\geq 17 \%)$, h-BN is no longer a layered structure and mechanical lift-off is impossible. These results are in agreement with 3 atom \% (19 atom \%) of $\mathrm{Al}$ concentrations of conventional (high-temperature) AlN/h-BN structures, as mentioned in the previous section, confirming the role of $\mathrm{Al}$ content in the mechanical lift-off. This study brings the unique approach of controlling the mechanical adhesion of this layered material, which can result in both lift-off controllable-layered h$\mathrm{BN}$ and robust h-BN layers.

If the hypothesis that lift-off is disabled by $\mathrm{Al}$ diffusion at high temperatures into the h-BN layer is correct, then it should be possible to have lift-off for high-temperature AlN buffer growth if the h-BN layer is sufficiently thick to prevent $\mathrm{Al}$ from diffusing completely through the h-BN layer. To test this, we grew the GaN/high-temperature/AlN/h-BN structure with an h-BN thickness of $25 \mathrm{~nm}$ (other growth conditions are kept the same) and then studied the mechanical lift-off of this set of samples. Figure 4 displays a camera photograph of the sample after lift-off, TEM and HAADF-STEM images, and EDX mapping for detailed structural analysis. In contrast to the $\mathrm{GaN} /$ high-temperature AlN/3 nm h-BN structures, the GaN/ high-temperature AlN/25 nm h-BN structures had been successfully lifted from the sapphire substrate. Identification of homogeneous and continuous h-BN layers comes from the HAADF-STEM FFT pattern (Figure 4a,b). The bright-field HAADF-STEM image (Figure 4c) shows a high contrast between h-BN, AlN layers and sapphire substrate. A quantitative analysis of various positions (green rectangles in Figure 4c) of this structure was done. The $\mathrm{Al}$ concentrations at the sapphire/h-BN interface and inside the h-BN layer are estimated at 2.0 atom \% (zone 1) and 1.2 atom \% (zone 2), respectively. However, at the h-BN/w-AlN interface up to 4 $\mathrm{nm}$, a high $\mathrm{Al}$ content was found with the estimation of 26.7
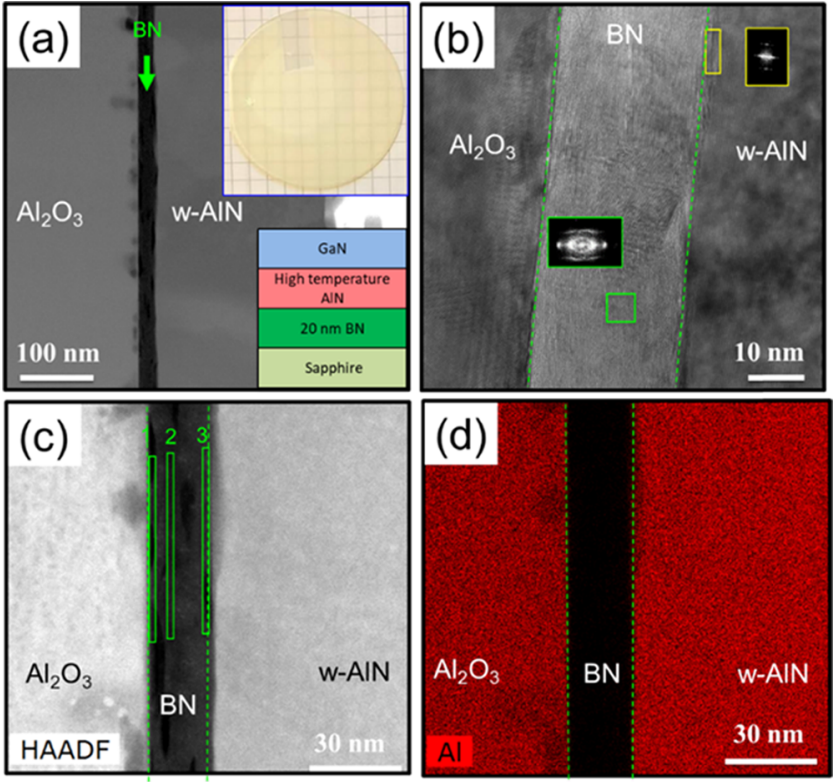

Figure 4. Cross-sectional high-resolution TEM and bright-field HAADF-STEM images, and the energy-dispersive X-ray spectroscopy (EDX) elemental mappings of $\mathrm{Al}$ of $\mathrm{GaN} /$ high-temperature $\mathrm{AlN}$ on $25 \mathrm{~nm}$ h-BN grown on sapphire, respectively. Inset: schematic illustration of the grown structure and the photograph of the sample after lift-off.

atom \% (zone 3). This large value of $\mathrm{Al}$ content indicates the presence of $\mathrm{Al}$ at this interface, confirming the limited diffusion of Al.

We first conclude that high-temperature growth directly affects Al diffusion, which plays an important role in the control of mechanical lift-off of the h-BN layer in particular and of III-nitride semiconductor in general. In addition, variation of the thickness of h-BN can open a new, flexible approach on the control of the lift-off process, expanding potential applications in mechanical transfer with the quality improvement of III-nitride device-based h-BN. These investigations can provide valuable insights into the remote control of the epitaxy growth of III-device-based h-BN using AlN as a buffer layer.

This understanding of the mechanism lift-off process further points out the way to avoid the h-BN delamination problem during growth and processing and to control mechanical lift-off of devices. With the intention of illustrating this, AlGaN/GaN HEMTs/high-temperature AlN/3 nm h-BN structures were grown. A set of these devices were subjected to mechanical liftoff, and we could not be able to accomplish it as seen in the inset of Figure 5a (left). Figure 5 displays the cross-sectional high-angle annular dark-field scanning transmission electron microscopy (HAADF-STEM) image and the energy-dispersive $\mathrm{X}$-ray spectroscopy (EDX) element mappings of $\mathrm{Al}$ of this sample, focusing on high-temperature AlN/h-BN/sapphire areas in the structure. The HAADF-STEM images (Figure 5a) clearly show the high contrast between sapphire substrate, h$\mathrm{BN}$, and AlN. Furthermore, at the sapphire/h-BN interface, we observed a $1-1.5 \mathrm{~nm}$ thick w-AlN layer. Besides, EDX element mappings of $\mathrm{Al}$ (Figure $5 \mathrm{~b}$ ) indicate the presence of $\mathrm{Al}$ in the $\mathrm{h}-\mathrm{BN}$ layer and at the h-BN/sapphire interface. The $\mathrm{Al}$ concentrations at different areas (green rectangles in Figure $5 b$ ) were estimated at around 26, 10, and 18 atom \%, corresponding to zones 1,2 , and 3 . The high value of $\mathrm{Al}$ 


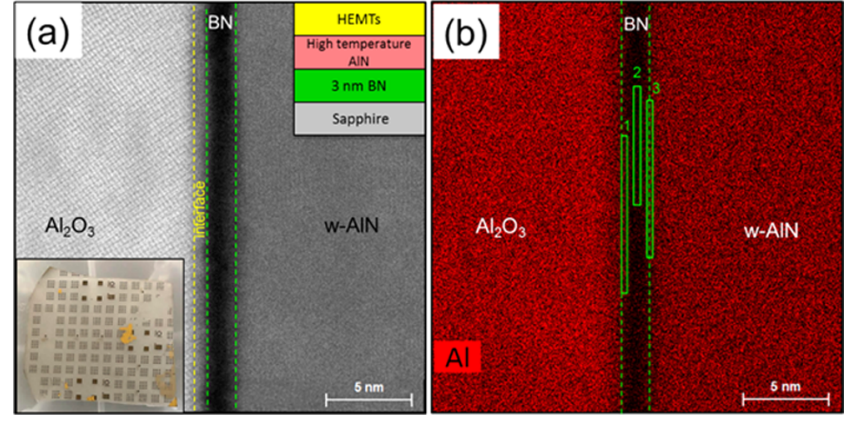

Figure 5. Cross-sectional bright-field HAADF-STEM image (a) and energy-dispersive X-ray spectroscopy (EDX) elemental mappings of $\mathrm{Al}$ (b) of HEMTs/high-temperature AlN/3 nm h-BN grown on sapphire. Inset in (a): schematic illustration of the grown structure photograph of the device after attempted lift-off.

concentration in the h-BN layer region is similar to the one of $\mathrm{GaN} /$ high-temperature AlN/3 nm h-BN, as presented above. Comparing with the study of the HEMTs device as reported earlier by Hiroki et al., ${ }^{2}$ this result is completely opposite. They grew and fabricated AlGaN/GaN HEMT on AlN (grown at a low temperature, $\sim 1000{ }^{\circ} \mathrm{C}$ ) on h-BN/sapphire substrates and successful lifted and transferred them from the host substrates to copper plates. This study further supports the important role of the growth temperature of AlN on the ability to control lift-off. Our investigation can open new perspectives in controlling and fabricating III-V devices grown on h-BN and further on other $2 \mathrm{D}$ materials. It can actually boost the device performance of heterostructures, bringing them close to industrial applications.

\section{CONCLUSIONS}

In conclusion, we have shown how to control the adhesion of 2D h-BN layers, which can result in either lift-off of layered h$\mathrm{BN}$ and devices grown on h-BN or mechanically inseparable robust $\mathrm{h}-\mathrm{BN}(\mathrm{Al})$ layers. We found that high-growth-temperature $\left(\sim 1280{ }^{\circ} \mathrm{C}\right) \mathrm{AlN}$ on h-BN leads to Al diffusion into h$\mathrm{BN}$, which enhances the surface and interface dangling bonds and anchors the layered h-BN. Detailed structural analyses of these samples were performed using TEM measurements to explore the lift-off mechanism. The effect of $\mathrm{Al}$ concentration into h-BN layers on the mechanical lift-off is verified by studying carefully BAlN alloys with different $\mathrm{Al}$ contents. It has been observed that for an $\mathrm{Al}$ content above $17 \%$, the layered BAlN sample cannot be lifted off, supporting our investigation. The control of the mechanical adhesion of different device structures such as HEMT-based h-BN layers has been examined to confirm the important role of $\mathrm{Al}$ diffusion for controlling the lift-off of the subsequent device structure grown on h-BN. These results reveal the perspective in understanding layered materials and controlling this layer-transfer technique. Moreover, they break through the blocked route to the largescale fabrication of III-V devices grown on h-BN, bringing them close to industrial applications.

\section{METHODS}

Sample Preparation. The growth was performed in an Aixtron MOVPE close-coupled showerhead (CCS) reactor on the (0001) sapphire substrate. Triethylboron (TEB), trimethylaluminum (TMAl), trimethylgallium (TMG), and ammonia $\left(\mathrm{NH}_{3}\right)$ were used as $\mathrm{B}, \mathrm{Al}, \mathrm{Ga}$, and $\mathrm{N}$ precursors, respectively. First, an h-BN layer (3 $\mathrm{nm}$ ) was grown on the sapphire substrate at $1280{ }^{\circ} \mathrm{C}$ and $90 \mathrm{mbar}$ pressure. Then, an AlN layer $(200 \mathrm{~nm})$ was grown directly on the $\mathrm{h}$ $\mathrm{BN}$ layer at high temperature $\left(1280^{\circ} \mathrm{C}\right)$ and low temperature $(1100$ ${ }^{\circ} \mathrm{C}$ ) to study the impact of AlN layer on mechanical lift-off under different growth conditions. A $300 \mathrm{~nm} \mathrm{GaN} \mathrm{layer} \mathrm{was} \mathrm{subsequently}$ grown on top of the AlN layer. In addition, to have a comprehensive understanding, the BAlN alloys were grown directly on the c-plane sapphire substrate with the variation of TMAl/III ratio. Detailed growth conditions of these heterostructures have been reported elsewhere. ${ }^{36}$ Finally, full heterostructures of AlGaN/GaN HEMTs were grown on the GaN/AlN/h-BN buffer for the mechanical lift-off final test. After growth, the transparent scotch was used to verify the mechanical lift-off possibility of all of the samples.

Characterizations. Scanning electron microscopy (SEM) and atomic force microscopy (AFM) were used to study the surface morphology of the samples before and after mechanical lift-off. For the crystallography studies, high-resolution transmission electron microscopy (HR-TEM) characterizations were performed on an aberration-corrected JEOL 2200FS electron transmission microscope. Prior to this study, cross-sectional lamellae were prepared using focused ion beam (FIB) thinning and ion milling after coating a 100 $\mathrm{nm}$ thick carbon for layer protection.

\section{ASSOCIATED CONTENT}

\section{Supporting Information}

The Supporting Information is available free of charge at https://pubs.acs.org/doi/10.1021/acsami.0c16850.

High-resolution X-ray diffraction (HR-XRD) and $\mathrm{Al}$ concentration depth profile (PDF)

\section{AUTHOR INFORMATION}

\section{Corresponding Author}

Abdallah Ougazzaden - Georgia Tech-CNRS, UMI 2958, Georgia Tech Lorraine, 57070 Metz, France; School of Electrical and Computer Engineering, Georgia Institute of Technology, Atlanta, Georgia 30332, United States; ○ orcid.org/0000-0002-9959-5280;

Email: abdallah.ougazzaden@georgiatech-metz.fr

\section{Authors}

Phuong Vuong - Georgia Tech-CNRS, UMI 2958, Georgia Tech Lorraine, 57070 Metz, France; (1) orcid.org/00000003-2775-7081

Suresh Sundaram - Georgia Tech-CNRS, UMI 2958, Georgia Tech Lorraine, 57070 Metz, France; School of Electrical and Computer Engineering, Georgia Institute of Technology, Atlanta, Georgia 30332, United States

Adama Mballo - Georgia Tech-CNRS, UMI 2958, Georgia Tech Lorraine, 57070 Metz, France

Gilles Patriarche - Centre de Nanosciences et de Nanotechnologies, Université Paris-Saclay, F-91460 Marcoussis, France

Stefano Leone - Fraunhofer IAF, Fraunhofer Institute for Applied Solid State Physics, 79108 Freiburg, Germany

Fouad Benkhelifa - Fraunhofer IAF, Fraunhofer Institute for Applied Solid State Physics, 79108 Freiburg, Germany

Soufiane Karrakchou - Georgia Tech-CNRS, UMI 2958, Georgia Tech Lorraine, 57070 Metz, France; School of Electrical and Computer Engineering, Georgia Institute of Technology, Atlanta, Georgia 30332, United States

Tarik Moudakir - Institut Lafayette, 57070 Metz, France

Simon Gautier - Institut Lafayette, 57070 Metz, France

Paul L. Voss - Georgia Tech-CNRS, UMI 2958, Georgia Tech Lorraine, 57070 Metz, France; School of Electrical and Computer Engineering, Georgia Institute of Technology, Atlanta, Georgia 30332, United States 
Jean-Paul Salvestrini - Georgia Tech-CNRS, UMI 2958, Georgia Tech Lorraine, 57070 Metz, France; School of Electrical and Computer Engineering, Georgia Institute of Technology, Atlanta, Georgia 30332, United States; (1) orcid.org/0000-0002-0482-1178

Complete contact information is available at: https://pubs.acs.org/10.1021/acsami.0c16850

\section{Author Contributions}

A.O. contributed to design the study and supervised the whole project. P.V. performed SEM, AFM, and mechanical lift-off; analyzed the results; and wrote the manuscript. S.S., P.V., and A.M. performed the epitaxial growth and prepared the $\mathrm{BN}$ samples. G.P. carried out HAADF-STEM experiments. S.L., F.B., S.S., T.M., and A.O. contributed with the growth and characterization of the $\mathrm{AlGaN} / \mathrm{GaN}$ HEMTs heterostructure on the GaN/AlN/h-BN buffer. S.K., S.G., and J.-P.S., were responsible for the processing of HEMTs heterostructure. All authors contributed to discussion of the results. All authors reviewed and commented on the manuscript.

\section{Notes}

The authors declare no competing financial interest.

All data generated or analyzed during this study are included in this published article.

\section{ACKNOWLEDGMENTS}

This study was partially funded by the French National Research Agency (ANR) under the GANEX Laboratory of Excellence (Labex). C. Manz and L. Kirste at Fraunhofer IAF are acknowledged for the characterization of the AlGaN/GaN HEMTs heterostructure on the GaN/AlN/h-BN buffer. The authors gratefully acknowledge Marcel Reis Soubkovsky (marcelsoubk.com) for his help in creating the Table of Contents (TOC) graphic.

\section{REFERENCES}

(1) Kobayashi, Y.; Kumakura, K.; Akasaka, T.; Makimoto, T. Layered Boron Nitride as a Release Layer for Mechanical Transfer of GaN-Based Devices. Nature 2012, 484, 223-227.

(2) Hiroki, M.; Kumakura, K.; Kobayashi, Y.; Akasaka, T.; Makimoto, T.; Yamamoto, H. Suppression of Self-Heating Effect in AlGaN/GaN High Electron Mobility Transistors by SubstrateTransfer Technology Using h-BN. Appl. Phys. Lett. 2014, 105, No. 193509.

(3) Ayari, T.; Sundaram, S.; Li, X.; El Gmili, Y.; Voss, P. L.; Salvestrini, J. P.; Ougazzaden, A. Wafer-Scale Controlled Exfoliation of Metal Organic Vapor Phase Epitaxy Grown InGaN/GaN Multi Quantum Well Structures Using Low-Tack Two-Dimensional Layered h-BN. Appl. Phys. Lett. 2016, 108, No. 171106.

(4) Ayari, T.; Bishop, C.; Jordan, M. B.; Sundaram, S.; Li, X.; Alam, S.; ElGmili, Y.; Patriarche, G.; Voss, P. L.; Salvestrini, J. P.; Ougazzaden, A. Gas Sensors Boosted by Two-Dimensional h-BN Enabled Transfer on Thin Substrate Foils: Towards Wearable and Portable Applications. Sci. Rep. 2017, 7, No. 15212.

(5) Motala, M. J.; Blanton, E. W.; Hilton, A.; Heller, E.; Muratore, C.; Burzynski, K.; Brown, J. L.; Chabak, K.; Durstock, M.; Snure, M.; Glavin, N. R. Transferrable AlGaN/GaN High-Electron Mobility Transistors to Arbitrary Substrates via a Two-Dimensional Boron Nitride Release Layer. ACS Appl. Mater. Interfaces 2020, 12, 2183721844.

(6) Glavin, N.; Chabak, K.; Heller, E.; Moore, E.; Prusnick, T.; Maruyama, B.; Walker, D.; Dorsey, D.; Paduano, Q.; Snure, M. Flexible Gallium Nitride for High-Performance, Strainable RadioFrequency Devices. Adv. Mater. 2017, 29, No. 1701838.
(7) Lee, J. S.; Choi, S. H.; Yun, S. J.; Kim, Y. I.; Boandoh, S.; Park, J.H.; Shin, B. G.; Ko, H.; Lee, S. H.; Kim, Y.-M.; Lee, Y. H.; Kim, K. K.; Kim, S. M. Wafer-Scale Single-Crystal Hexagonal Boron Nitride Film via Self-Collimated Grain Formation. Science 2018, 362, 817-821.

(8) Liang, D.; Wei, T.; Wang, J.; Li, J. Quasi van Der Waals Epitaxy Nitride Materials and Devices on Two Dimension Materials. Nano Energy 2020, 69, No. 104463.

(9) Gautier, S.; Moudakir, T.; Patriarche, G.; Rogers, D. J.; Sandana, V. E.; Téherani, F. H.; Bove, P.; El Gmili, Y.; Pantzas, K.; Sundaram, S.; Troadec, D.; Voss, P. L.; Razeghi, M.; Ougazzaden, A. Structural and Compositional Characterization of MOVPE GaN Thin Films Transferred from Sapphire to Glass Substrates Using Chemical Liftoff and Room Temperature Direct Wafer Bonding and GaN Wafer Scale MOVPE Growth on ZnO-Buffered Sapphire. J. Cryst. Growth 2013, 370, 63-67.

(10) Kelly, M. K.; Vaudo, R. P.; Phanse, V. M.; Görgens, L.; Ambacher, O.; Stutzmann, M. Large Free-Standing GaN Substrates by Hydride Vapor Phase Epitaxy and Laser-Induced Liftoff. Jpn. J. Appl. Phys. 1999, 38, L217-L219.

(11) Caldwell, J. D.; Aharonovich, I.; Cassabois, G.; Edgar, J. H.; Gil, B.; Basov, D. N. Photonics with Hexagonal Boron Nitride. Nat. Rev. Mater. 2019, 4, 552-567.

(12) Li, Y.; Garnier, V.; Steyer, P.; Journet, C.; Toury, B. MillimeterScale Hexagonal Boron Nitride Single Crystals for Nanosheet Generation. ACS Appl. Nano Mater. 2020, 3, 1508-1515.

(13) Ahmed, K.; Dahal, R.; Weltz, A.; Lu, J. J.-Q.; Danon, Y.; Bhat, I. B. Solid-State Neutron Detectors Based on Thickness Scalable Hexagonal Boron Nitride. Appl. Phys. Lett. 2017, 110, No. 023503.

(14) McGregor, D. S.; Unruh, T. C.; McNeil, W. J. Thermal Neutron Detection with Pyrolytic Boron Nitride. Nucl. Instrum. Methods Phys. Res., Sect. A 2008, 591, 530-533.

(15) Bae, S.-H.; Kum, H.; Kong, W.; Kim, Y.; Choi, C.; Lee, B.; Lin, P.; Park, Y.; Kim, J. Integration of Bulk Materials with TwoDimensional Materials for Physical Coupling and Applications. Nat. Mater. 2019, 18, 550-560.

(16) Journot, T.; Bouchiat, V.; Gayral, B.; Dijon, J.; Hyot, B. SelfAssembled UV Photodetector Made by Direct Epitaxial GaN Growth on Graphene. ACS Appl. Mater. Interfaces 2018, 10, 18857-18862.

(17) Snure, M.; Siegel, G.; Look, D. C.; Paduano, Q. GaN and AlGaN/GaN Heterostructures Grown on Two Dimensional BN Templates. J. Cryst. Growth 2017, 464, 168-174.

(18) Ayari, T.; Sundaram, S.; Bishop, C.; Mballo, A.; Vuong, P.; Halfaya, Y.; Karrakchou, S.; Gautier, S.; Voss, P. L.; Salvestrini, J. P.; Ougazzaden, A. Novel Scalable Transfer Approach for Discrete IIINitride Devices Using Wafer-Scale Patterned h-BN/Sapphire Substrate for Pick-and-Place Applications. Adv. Mater. Technol. 2019, 4, No. 1900164.

(19) Chugh, D.; Wong-Leung, J.; Li, L.; Lysevych, M.; Tan, H. H.; Jagadish, C. Flow Modulation Epitaxy of Hexagonal Boron Nitride. 2D Mater. 2018, 5, No. 045018.

(20) Bera, K.; Chugh, D.; Patra, A.; Tan, H.; Roy, C. Strain Distribution and Thermal Strain Relaxation in MOVPE Grown HBN Films on Sapphire Substrates. 2019. arXiv.orge-Printarchive.https:// arxiv.org/pdf/1907.05591.pdf.

(21) Liu, L.; Edgar, J. H. Substrates for Gallium Nitride Epitaxy. Mater. Sci. Eng., R 2002, 37, 61-127.

(22) Kim, J.; Bayram, C.; Park, H.; Cheng, C.-W.; Dimitrakopoulos, C.; Ott, J. A.; Reuter, K. B.; Bedell, S. W.; Sadana, D. K. Principle of Direct van Der Waals Epitaxy of Single-Crystalline Films on Epitaxial Graphene. Nat. Commun. 2014, 5, No. 4836.

(23) Sundaram, S.; Li, X.; Alam, S.; Ayari, T.; Halfaya, Y.; Patriarche, G.; Voss, P. L.; Salvestrini, J. P.; Ougazzaden, A. MOVPE van Der Waals Epitaxial Growth of AlGaN/AlGaN Multiple Quantum Well Structures with Deep UV Emission on Large Scale 2D h-BN Buffered Sapphire Substrates. J. Cryst. Growth 2019, 507, 352-356.

(24) Wu, Q.; Yan, J.; Zhang, L.; Chen, X.; Wei, T.; Li, Y.; Liu, Z.; Wei, X.; Zhang, Y.; Wang, J.; Li, J. Growth Mechanism of AlN on Hexagonal BN/Sapphire Substrate by Metal-Organic Chemical Vapor Deposition. CrystEngComm 2017, 19, 5849-5856. 
(25) Chugh, D.; Adhikari, S.; Wong-Leung, J.; Lysevych, M.; Jagadish, C.; Tan, H. H. Improving the Morphology and Crystal Quality of AlN Grown on Two-Dimensional HBN. Cryst. Growth Des. 2020, 20, 1811-1819.

(26) Wu, Q.; Yan, J.; Zhang, L.; Chen, X.; Wei, T.; Li, Y.; Liu, Z.; Wei, X.; Zhang, Y.; Wang, J.; Li, J. Growth Mechanism of AlN on Hexagonal BN/Sapphire Substrate by Metal-Organic Chemical Vapor Deposition. CrystEngComm 2017, 19, 5849-5856.

(27) Sundaram, S.; Li, X.; Halfaya, Y.; Ayari, T.; Patriarche, G.; Bishop, C.; Alam, S.; Gautier, S.; Voss, P. L.; Salvestrini, J. P.; Ougazzaden, A. Large-Area van Der Waals Epitaxial Growth of Vertical III-Nitride Nanodevice Structures on Layered Boron Nitride. Adv. Mater. Interfaces 2019, 6, No. 1900207.

(28) Kobayashi, Y.; Akasaka, T. Hexagonal BN Epitaxial Growth on (0001) Sapphire Substrate by MOVPE. J. Cryst. Growth 2008, 310, 5044-5047.

(29) Nilsson, D.; Janzén, E.; Kakanakova-Georgieva, A. Lattice Parameters of AlN Bulk, Homoepitaxial and Heteroepitaxial Material. J. Phys. D: Appl. Phys. 2016, 49, No. 175108.

(30) Matsumoto, K.; Ono, T.; Honda, Y.; Torigoe, K.; Kushimoto, M.; Amano, H. Origin of Acceptor Diffusion into Silicon Substrate during GaN Growth by Metal Organic Chemical Vapor Deposition. Jpn. J. Appl. Phys. 2019, 58, No. 075502.

(31) Kumagai, Y.; Tajima, J.; Ishizuki, M.; Nagashima, T.; Murakami, H.; Takada, K.; Koukitu, A. Self-Separation of a Thick AlN Layer from a Sapphire Substrate via Interfacial Voids Formed by the Decomposition of Sapphire. Appl. Phys. Express 2008, 1, No. 045003 .

(32) Chaaben, N.; Laifi, J.; Bouazizi, H.; Saidi, C.; Bchetnia, A.; El Jani, B. Study of Al Diffusion in GaN during Metal Organic Vapor Phase Epitaxy of AlGaN/GaN and AlN/GaN Structures. Mater. Sci. Semicond. Process. 2016, 42, 359-363.

(33) Cai, D.; Chen, X.; Xu, H.; Lin, N.; Xu, F.; Chen, H. Abruptness Improvement of the Interfaces of $\mathrm{AlGaN} / \mathrm{GaN}$ Superlattices by Cancelling Asymmetric Diffusion. Jpn. J. Appl. Phys. 2013, 52, No. 08JB30.

(34) Haneda, H.; Ohgaki, T.; Sakaguchi, I.; Ryoken, H.; Ohashi, N.; Yasumori, A. SIMS Analysis of Impurities and Nitrogen Isotopes in Gallium Nitride Thin Films. Appl. Surf. Sci. 2006, 252, 7265-7268.

(35) Yang, X.; Nitta, S.; Pristovsek, M.; Liu, Y.; Nagamatsu, K.; Kushimoto, M.; Honda, Y.; Amano, H. Interface Amorphization in Hexagonal Boron Nitride Films on Sapphire Substrate Grown by Metalorganic Vapor Phase Epitaxy. Appl. Phys. Express 2018, 11, No. 051002 .

(36) Vuong, P.; Mballo, A.; Sundaram, S.; Patriarche, G.; Halfaya, Y.; Karrakchou, S.; Srivastava, A.; Krishnan, K.; Sama, N. Y.; Ayari, T.; Gautier, S.; Voss, P. L.; Salvestrini, J. P.; Ougazzaden, A. Single Crystalline Boron Rich B(Al)N Alloys Grown by MOVPE. Appl. Phys. Lett. 2020, 116, No. 042101.

(37) Li, X.; Sundaram, S.; El Gmili, Y.; Ayari, T.; Puybaret, R.; Patriarche, G.; Voss, P. L.; Salvestrini, J. P.; Ougazzaden, A. LargeArea Two-Dimensional Layered Hexagonal Boron Nitride Grown on Sapphire by Metalorganic Vapor Phase Epitaxy. Cryst. Growth Des. 2016, 16, 3409-3415. 